\title{
Rats, nets, maps, and the emergence of place cells
}

\author{
ALESSANDRO TREVES \\ University of Oxford, Oxford, England \\ and \\ ORAZIO MIGLINO and DOMENICO PARISI \\ Istituto di Psicologia, Consiglio Nazionale delle Ricerche, Rome, Italy
}

\begin{abstract}
We study through computer simulations the motion in space of small networks consisting of a few sensory, intermediate, and motor units linked by feedforward connections of initially random strengths. Evolutionary pressure, exerted through random differentiation and selective reproduction, can force such objects to adapt to perform elementary navigation tasks similar to those used in investigating hippocampal function in rats. The connection strengths resulting from the adaptation process are shown to provide intermediate units with response characteristics similar to those of place cells found in the rat hippocampus. These results illustrate the ease with which "place" units emerge in any minimal circuitry geared to solve simple navigation tasks, and highlight the importance of considering the complexity of the memory performance required, rather than the relatively trivial spatial "computations" involved, while using those tasks to explore hippocampal structure and function.
\end{abstract}

The finding of place cells in the rat hippocampus (O'Keefe, 1976; O'Keefe \& Dostrovsky, 1971) has largely inspired the modern formulation (O'Keefe \& Nadel, 1978) of the cognitive map theory (Tolman, 1948), which in turn has been very influential in shaping hippocampal research over the last 15 years (see, e.g., the debate in Nadel, 1991). The need to test the specific predictions of the theory has stimulated the development of experimental apparatuses and tasks-including the water maze (Morris, 1984) and the circular platform (Barnes, 1979) - which allow manipulation of the spatial memory demands imposed on the animals, in conjunction with lesions, pharmacological interventions, or cell recording. When stripped of their memory components, these tasks appear very simple in terms of mere spatial navigation. In both cases mentioned above, for example, the rat has just to reach one particular final location from an initial one, in a circular environment, on the basis of external cues. It is precisely this simplicity, of course, which makes these laboratory setups, in contrast to more "ecological" situations, ideal for manipulations concerning memory.

While most current attempts to understand the structure and functional operation of the hippocampus (Brown $\&$ Zador, 1990) revolve around its suspected memory role (McNaughton \& Nadel, 1990; Rolls, 1989), largely along the lines followed by Marr 20 years ago (Marr, 1971),

\footnotetext{
We are indebted to Franca Tecchio, who developed part of the software, and to Shane O'Mara, who made quite frank comments. A.T. gratefully acknowledges the hospitality of the Istituto di Psicologia, where the work originated. Correspondence should be addressed to A. Treves, Department of Experimental Psychology, University of Oxford, South Parks Rd., Oxford OX1 3UD, U.K. (Telephone, 44-865-271419; Fax, 44-865-310447; e-mail, treves@vax.ox.ac.uk).
}

computational models have also been proposed that aim at elucidating the actual spatial information processing ascribed to the hippocampus (O'Keefe, 1990; Schmajuk, 1990; Zipser, 1985). These models view the processing associated with navigation as an algorithmic sequence of transformations of the sensory input, which result, at an intermediate stage, in information about space being coded in a set of units that constitute an (allocentric) map of the environment - the place cells. What are described in detail are then the computations which allow the emergence of unit responses coding for place. It should pertaps be noted that the notion of "place cells" comes out more clearly from such theoretical constructs than from experimental data. The latter (McNaughton, Barnes, \& O'Keefe, 1983) show, indeed, that most rat hippocampal neurons respond, while the animal is performing a spatial memory task, in a way strongly correlated with the animal's location. The responses, though, are generally reported to bear strong correlations also with several other variables, including, for example, the direction the animal is facing (or its local view), with fewer data (O'Keefe, 1979) supporting the notion of pure, orientation-independent place cells.

We address the question of the spatial computations involved in performing a navigation task in a different way. We consider a population of tiny three-layer networks (Parisi, Cecconi, \& Nolfi, 1990), with the first layer units coding for sensory input and the third layer ones for motor output. Feedforward connections with random strengths, and the activity of units in one layer, determine the activity of units in the next layer. Each network is placed a few times in a circular environment with some external landmarks. Part of the population then undergoes replication with random differentiation (at the connection strengths level), the replicating individuals being selected 
(Goldberg, 1989) on the basis of their ability to reach a given location in the environment. The networks are passed no information relative to the goal, nor do they know that it is "good" for them to get there-they have no sense of purpose, so to speak; and, in fact, those of the first generations tend to stay put, or move aimlessly in a stereotyped pattern until they hit the boundary, or appear to toddle around, subject to the random modulation of their motor commands. Evolutionary adaptation (Holland, 1975), however, eventually produces a tendency to move towards the goal, and later generations aim for it independently of their (randomly chosen) starting position.

We call these networks randomly adapted toddlershenceforth RATs, for the sake of brevity. In their utter simplicity, they provide a tool for studying the computational demands associated with spatial memory tasks, and which actual rats have to meet on top of the memory demands. In particular, we discuss here the emergence of "place"' units among the intermediate units of a RAT, and its implications on the appraisal of rat hippocampal data.

\section{METHOD}

A RAT of the standard strain consists of 9 units in the sensory layer, 16 in the intermediate, and 2 in the motor layer. There are 8 visual sensory units, pairs of which code for the presence, within their receptive fields, of each of 4 external landmarks, or cues. The 2 units of a pair correspond to the right and left eye, and their receptive fields extend $0^{\circ}-120^{\circ}$ on each side, with respect to the direction the RAT is facing. If the relative cue is inside the receptive field of 1 unit, its activity $a$ is proportional to the perceived size of the cue, that is, decreasing with increasing distance between the RAT and the cue; if the cue is outside the receptive field, $a=0$. In addition, there is 1 somatosensory unit, which is active only when the RAT has its nose against the wall-the boundary of the circular environment.

The activity of each intermediate unit is determined by those of the sensory units as

$$
a_{i}=\max \left(\sum_{s} w_{i, s} a_{s}, 0\right),
$$

where $w_{i, s}$ denotes a connection strength. Intermediate units determine in the same fashion the activity of motor units, with the only difference being that this is now cut off at the maximal level $a_{\max }=1$, so that RATs do not exceed a maximal speed. Motion is coded in the activities $a_{1}, a_{2}$ of the 2 motor units in the following way: if $D_{\text {max }}$ is the maximal displacement in a time unit, the RAT moves forward by an amount $D f=D_{\max } \times\left(a_{1}+a_{2}\right) / 2$, turns around by an angle $D \alpha=90^{\circ} \times\left(a_{1}-a_{2}\right)$ and moves laterally, left or right depending on the sign, by an amount $D s=D f \times D \alpha / 2$. It is not allowed to go beyond the circular walls.

Physical dimensions are obviously arbitrary; for concreteness, they are expressed in units such as centimeters or seconds, and chosen so as to imitate those of the water maze paradigm (Morris, 1984). Thus, RATs are placed at random locations (facing inward) along the border of a "pool" $214 \mathrm{~cm}$ in diameter, containing in a fixed location in one of its quadrants a "platform" $9 \mathrm{~cm}$ in diameter. The four landmarks are located around the pool $150 \mathrm{~cm}$ from its center, as in the figures below. Time is discretized in units of half a second, approximately four times the period of the rat $\theta$ rhythm (cf. Sharp, 1991). $D_{\max }$ is set to $12 \mathrm{~cm}$ so that RATs can move around the pool at the maximal speed of $24 \mathrm{~cm} / \mathrm{sec}$. They are removed once they reach the platform or after $120 \mathrm{sec}$, which- ever happens first, and the escape latency is recorded for each of 10 trials. In some of the experiments, two circular environments are used, differing in the location, relative to the platform, of the extramaze cues. In this case, 10 trials are run in each environment.

The first generation of 100 RATs has random connection strengths, each connection strength being drawn from a flat distribution between -1.0 and 1.0. After the trials, the 20 RATs of each generation who achieved the least cumulative latencies are allowed to replicate 5 times each to produce the 100 offsprings of the new generation. These inherit the connection strengths of their parent except for the addition, at $20 \%$ of the connections, of a random number, again with the same distribution. The whole experiment has been run several times on IBM 486 workstations and on SUN SPARCstations SLC, on which a second of RAT motion takes approximately $0.002 \mathrm{sec}$ of CPU time.
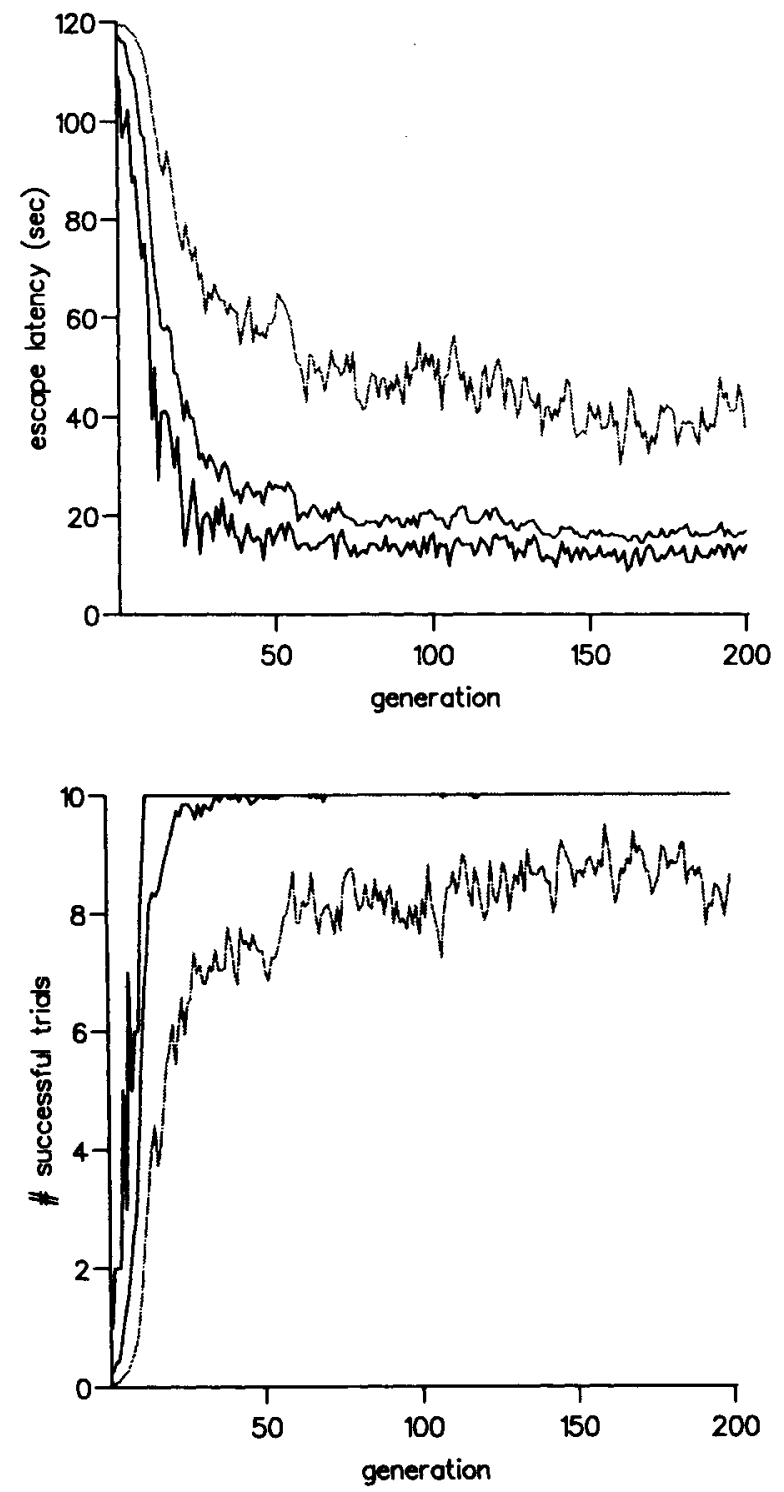

Figure 1. Mean escape latency (top) and mean number of successful trials (bottom) for the single best, the 20 best, and the whole population vs. generation. 


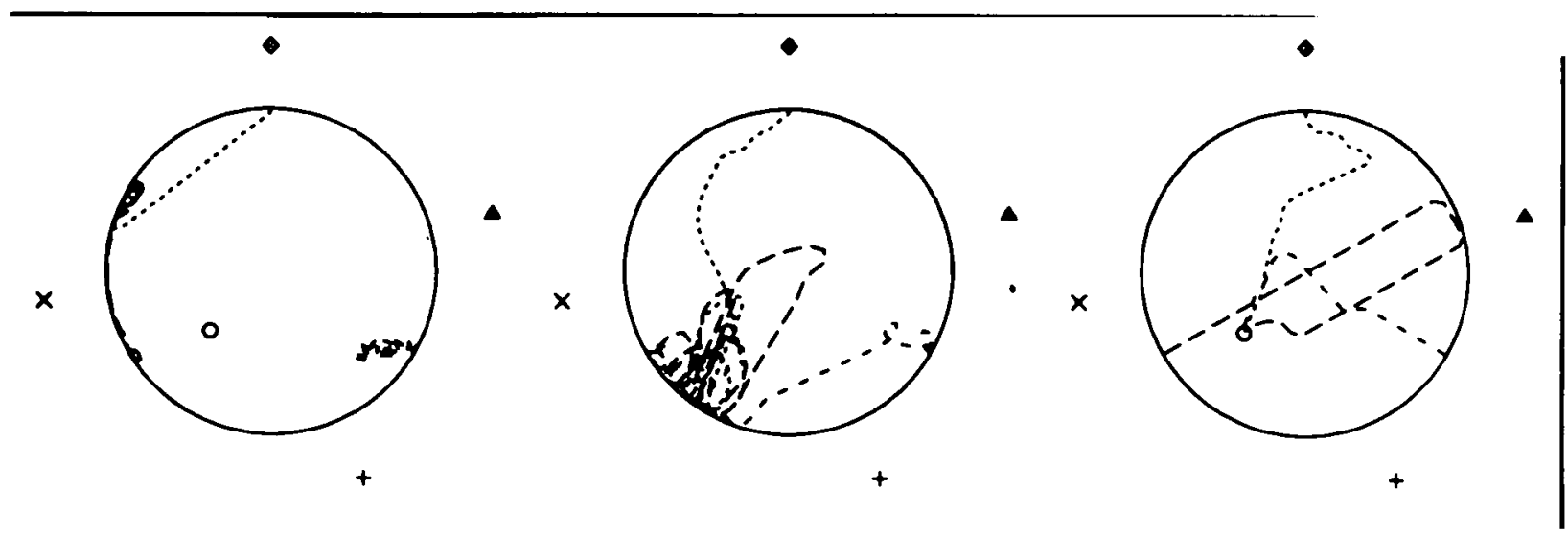

Figure 2. Trajectories taken in the test trials by the best RATs of the 1st, 10th, and 100th generation. The extramaze cues are located as indicated by the symbols. It is amusing to note that RATs' trajectories and escape latencies are more remiscent of those characterting hippocampectomized rats than of those typical of control animals (Morris, Garrud, Rawlins, \& O'Keefe, 1982).

\section{RESULTS}

\section{Behavior}

RATs "learn," on a phylogenetic scale, to reach the platform. This happens gradually, with the first few, being among those that happen to be endowed with connections that make them move, stumbling onto the platform once or twice in their set of trials. The evolutionarily advantageous tendency to move quickly diffuses across populations, as subsequently do the tendencies to turn around upon bouncing against the wall, and to move in the correct general direction, until RATs of later generations aim for the goal from whatever direction they are released. The result of a typical experiment (run in a single environment) is illustrated in Figure 1, which shows how the mean escape latency decreases from generation to generation, and how the number of successful trials (when the platform is actually reached within the $120 \mathrm{sec}$ ) rapidly increases.

To exemplify the kind of motor patterns that emerge, the best performing RATs of each generation are kept, and are made to run three further 60-sec trials (from starting points at $120^{\circ}$ of each other), during which a record is kept of each trajectory. Figure 2 shows the trajectories taken by the best individual of the 1st, 10th, and 100th generation.

In another set of experiments, we placed each RAT in two enviroments, using the same set of four extramaze cues, but placed in different locations. RATs adapt to reach the platform in each of the two situations, thus acquiring a capability to discriminate between two possible relative arrangements of the same landmarks, and to solve each navigation task accordingly. It takes them, though, a somewhat longer time to adapt to perform successfully, and even in the long-term performances tend to be poorer. This is illustrated in Figure 3 and in Figure 4, which shows the trajectories taken in both environments by a RAT of a late generation.

To test the robustness of these findings, we have run several experiments with RATs of different strains, vary-
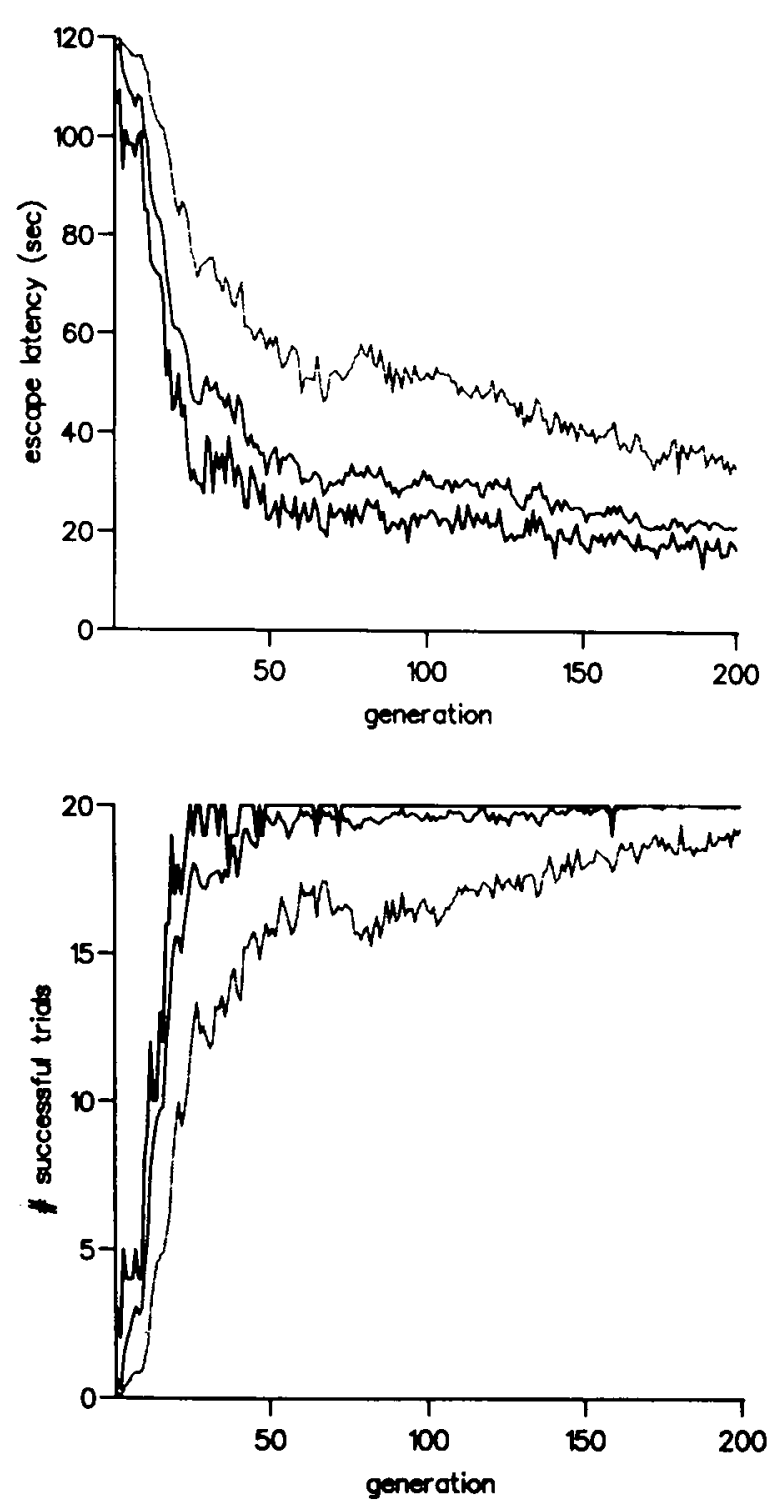

Figure 3. Same as Figure 1, for a two-environments experiment. 




Figure 4. Trajectories taken in the test trials by the best RAT of the 200th generation, in each of the two environments in which this experiment was run. Notice the different locations of the cues in the two environments. Interestingly, in one of the trials in the bottom environment, the RAT seems to search for the platform in the location where it would be, relative to the two nearest cues, in the top environment.

ing, for example, the maximal speed, the size of the receptive fields, or the number of intermediate units. The rather bland differences that result are all in the obvious directions-for example, poorer performances with fewer units-and overall the standard strain data is representative qualitatively if not quantitatively.

\section{Single Unit Responses}

Place units emerge among the intermediate units of successfully performing RATs. The responses of most of these units, while being dependent on the location of the RAT in the environment, are also strongly dependent on its orientation, but occasionally units appear whose responses do not vary much with orientation. These findings are exemplified in Figures 5 and 6 . The best RAT of the last generation has been placed on a grid of locations covering all the circular environment, in the four orientations NW, SW, SE, NE, and the activity of each of the 16 intermediate units has been recorded. Figure 5 shows the activity, averaged over the four orientations, of all 16 intermediate units. Clearly position in space is an important determinant of the unit responses. These, however, appear to vary markedly with orientation, with a single unit displaying a "place field" with some degree of constancy across orientations. Figure 6 shows the response levels of that unit in the four orientations sampled, together with the responses of another unit whose place field is strongly orientation dependent.

The number of intermediate units whose responses are both strongly place dependent, and relatively invariant for different orientations of the RAT, varies greatly from experiment to experiment, as it appears that quite different computational strategies are selected by different runs of the evolution process. The tendency to produce such units is enhanced, though, in populations of RATs that adapt to reach the platform in two different environments. Figure 7 shows the unit responses of one such RAT, again averaged over the four orientations, and in Figure 8 the responses of two units which display some degree of orientation invariance is split up over different orientations and different environments. These two units respond in similar ways to very different configurations of sensory stimuli, whose common denominator is that they define a portion of space (in one case, of boundary) relative to the location of the goal, and we would argue that similar response curves, recorded in the rat, would be classified as characterizing genuine place units.

\section{DISCUSSION}

RATs are simple creatures, and the rapidity with which they adapt to reach the goal demonstrates, first of all, how easy it is to perform the task. This is a very desirable property of experimental paradigms designated to test spatial memory in real rats, the idea being that of dissociating memory, as far as possible, from the purely sensorimotor aspects that interact with it in navigation.

Secondly, units with responses similar to those of "place" cells emerge in RATs in the absence of any specific network architecture, of any peculiar organization of the connections, or of any activity-dependent plasticity. The emergence of such units is to be regarded as nothing more than an element of some of the solutions that a random adaptive process finds to a simple spatial task. This type of solution seems to be particularly important when the task involves reaching a platform in more than one fixed environment (as defined by the location of extramaze cues). Nothing, of course, prevents one from saying that those place units represent "cognition" of space, nor from debating to what extent they constitute an allocentric rather than an egocentric system, although it is entirely possible that such terms will be less comfortably used in the case of RATs. A more constructive approach is to attempt to quantify the representational demands made, for example, by the multiplicity of sensory constellations present in several different environments, and to analyze how they can be met by particular coding schemes. A third approach uses "neural network" modeling.

In a recent interesting article, Sharp (1991) describes a layered network which is exposed to sensory stimuli sim- 
ABOVE 12

$11-12$

$10-11$

$9-10$

$8-9$

$7-8$

$6-7$

$5-6$

$4-5$

$3-4$

$2-3$

BELOW

2
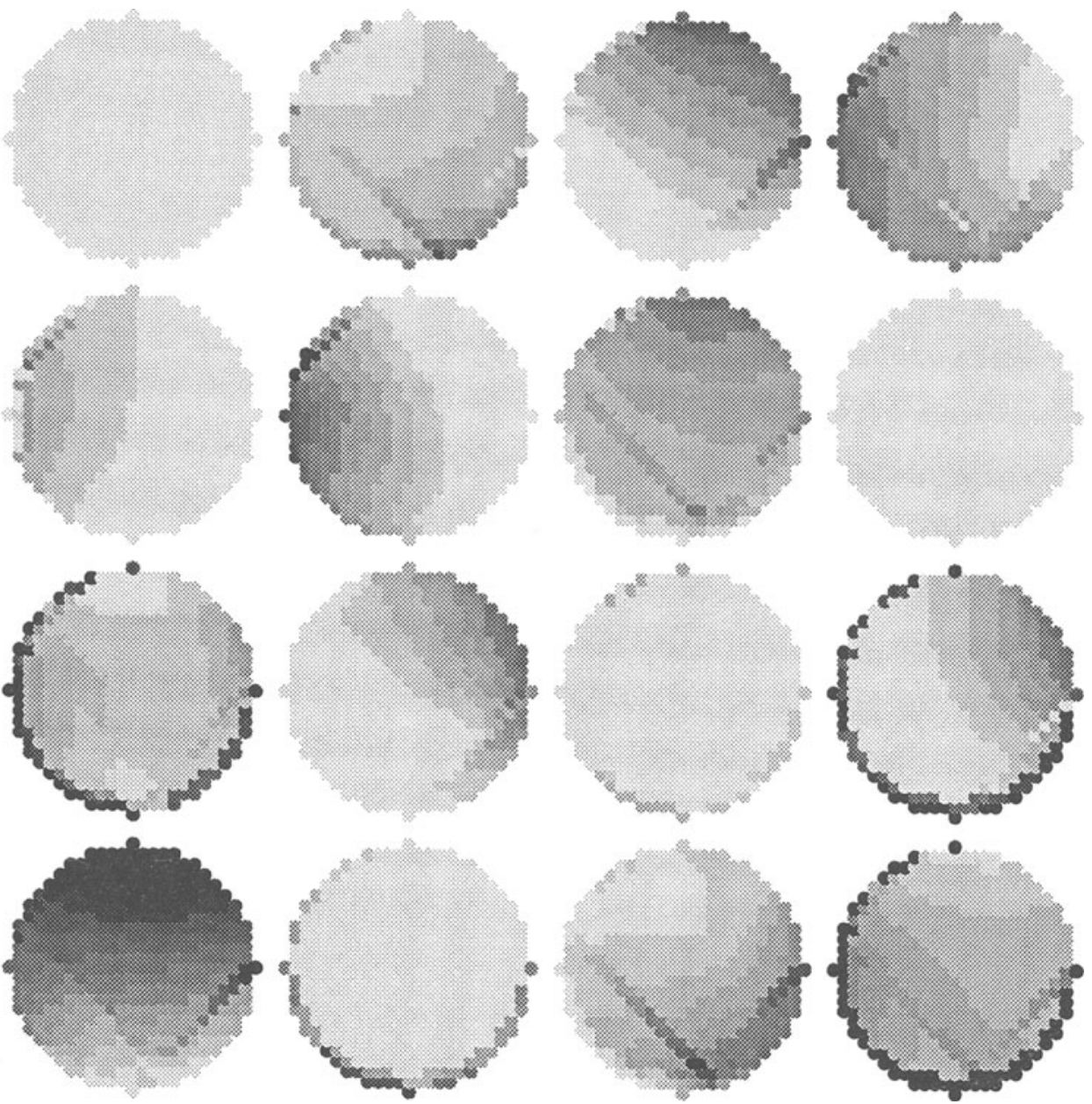

Figure 5. Intensity of intermediate unit responses, coded as in the legend, averaged over four orientations. In comparing these responses with those recorded in rats (not in the water maze, but, e.g., in the dry circular environment of Sharp, Kubie, \& Muller, 1990), it should be noted that the full orientation average makes the RAT data appear less concentrated in one portion of space than it would be if only the orientations along each actual trajectory were included.

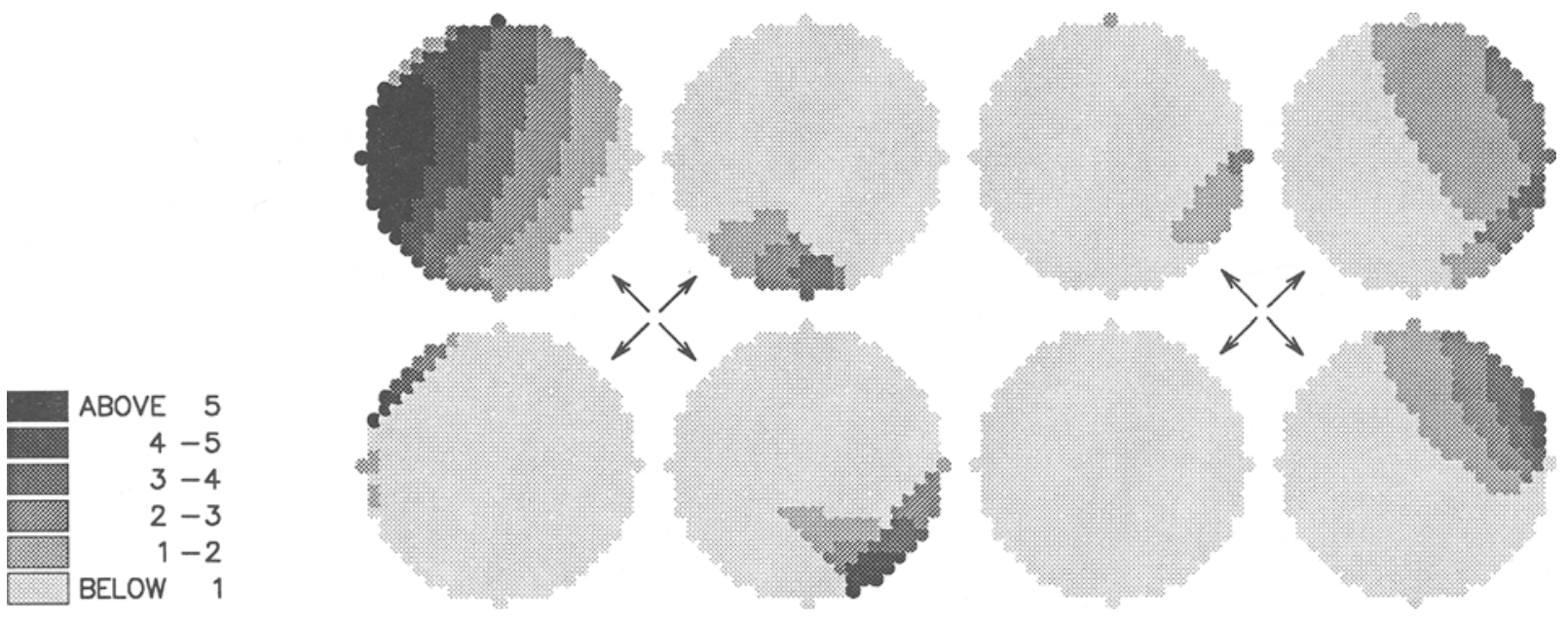

Figure 6. The responses, in each orientation indicated by the arrows, for the far right unit of the top row of Figure 5 (left)-exhibiting a strong orientation dependency - and for the second unit from the left of the third row (right)-the one varying the least with orientation. 

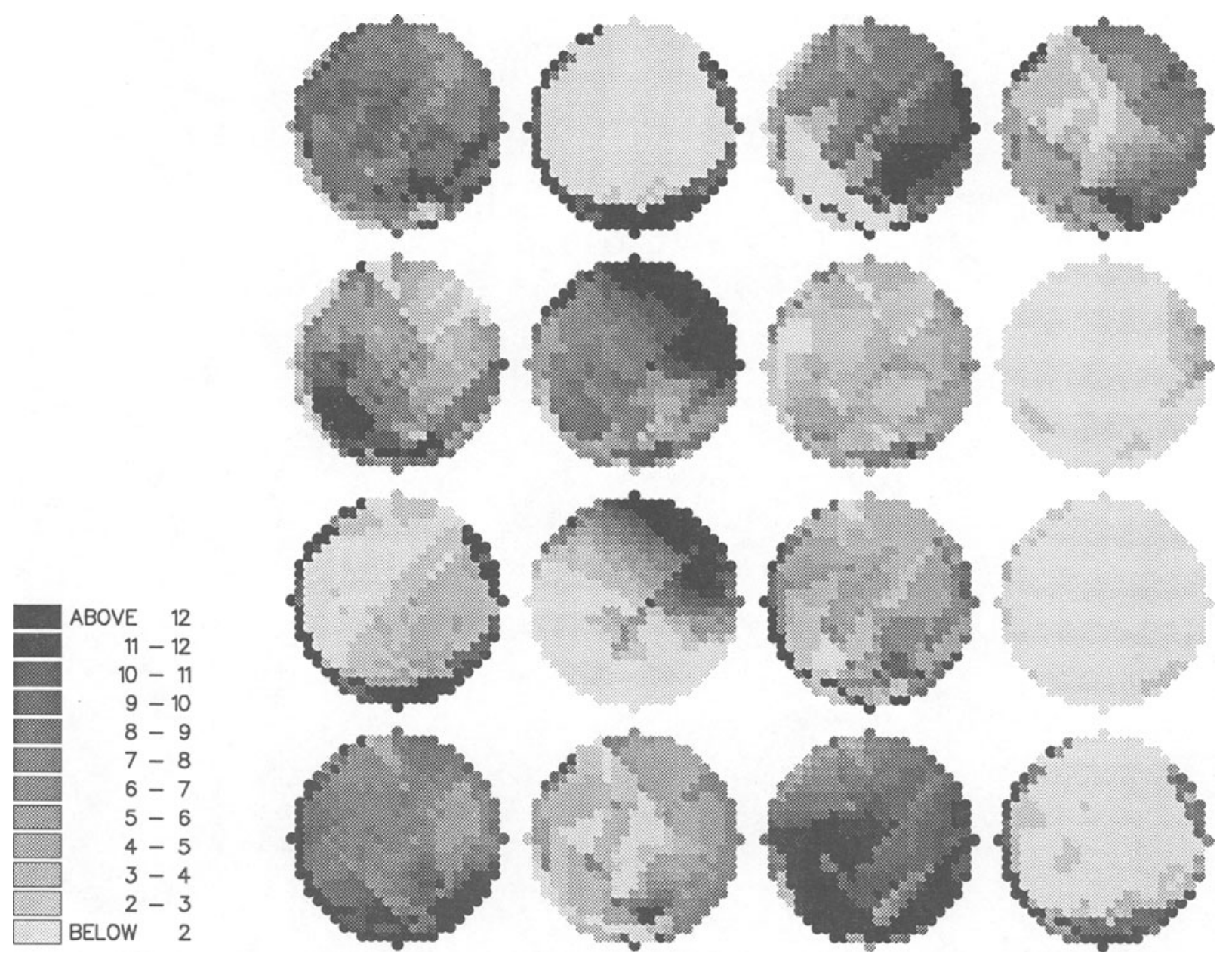

Figure 7. Same as Figure 5, for a RAT adapted to two different sets of cue locations.

ilar to those experienced by a rat performing spatial memory tasks. Again, the network develops units with a response similar to those of place cells in the rat. Sharp relates this fact to the competitive architecture (Rumelhart \& Zipser, 1986) of her model and the Hebbian plasticity of its connections, but she also warns against deriving too strong inferences from this type of neural network modeling. We would like to reiterate her warning. With all due differences (e.g., the phylogenetic rather than ontogenetic scale), experiments with RATs suggest that the emergence of place cells has much more to do with the experimental paradigm than with the details of the neural or pseudoneural circuitry involved.

One may also argue that the connections resulting from our "evolutionary" procedure are equivalent to, say, those produced by a particular learning rule, and maybe go on to analyze the transformation they implement, or even consider how such transformation could be realized by hippocampal cells. Can such an analysis tell us much about the organization of the hippocampus? The hippocampus of a rat contains more that $10^{6}$ cells (not to mention that of primates), displaying a remarkably structured organization. It seems very unlikely that we can make sense of those circuits by focusing solely on behavioral correlates that tiny amorphous networks of 27 units also produce after a few random attempts. The indication then, which RAT experiments per absurdum may help substantiate, is that only by carefully considering the whole spectrum of computational demands associated with solving a class of tasks-in particular the memory component (Treves \& Rolls, in press)-not just the mere information processing, it will be possible to infer constraints on the circuitry required, and thus attempt to understand how hippocampal networks operate. 




Figure 8. The responses in each orientation and each environment (top and bottom parts), for the second unit from the left of the third row of Figure 7 (left) and the far right unit of the fourth row (right). The latter could be termed a "boundary" unit, and its response appears to be modulated to a great extent by the somatosensory unit; note, though, that this unit shows a clear preference for the bottom and lef parts of the boundary, in both environments.

\section{REFERENCES}

BARNes, C. A. (1979). Memory deficits associated with senescence: A neurophysiological and behavioural study in the rat Journal of Comparative Physiology, 93, 74-104

Brown, T. H., \& ZADOR, A. (1990). Hippocampus. In G. M. Shepherd (Ed.), The synaptic organization of the brain (pp. 346-388). New York: Oxford University Press.

GoldBerG, D. E. (1989). Genetic algorithms in search, optimization and machine learming, Reding, MA: Addison-Wesley.

Holland, J. H. (1975). Adaptation in natural and artificial systems. Ann Arbor, University of Michigan Press.

MArR, D. (1971). Simple memory: A theory for anchicortex. Philosophical Transactions of the Royal Society (London), Series B, 262, 24-81.

McNaughton, B. L., Barnes, C. A., O'Keefe, J. (1983). The contribution of position, direction and velocity to single unit activity in the hippocampus of freely-moving rat. Experimental Brain Research, 52, 41-49.

McNaughton, B. L., * NADEL, L., (1990). Hebb-Marr networks and the neurobiological representation of action in space. In $\mathrm{M}$. A. Gluck \& D. E. Rumelhart (Eds.), Neuroscience and connectionist theory (pp. 1-63). Hillsdale, NJ: Erlbaum
MoRRIs, R. G. M. (1984). Developments of a water-maze procedure for studying spatial learning in the rat. Joumal of Neuroscience Methods, 11, 47-60.

Morris, R. G. M., Garrud, P., Rawlins, R. N. P., * OKeefe, J. (1982). Place navigation impaired in rats with hippocampal lesions. Nature, 297, 681-683.

NADEL, L. (1991). Forum: Is the hippocampal formation preferentially involved in spatial behaviour? Hippocampus, 1 (3), 221-292.

OKEeFE, J. (1976). Place units in the hippocampus of the frecly-moving rat. Experimental Neurology, 51, 78-109.

O'Keefe, J. (1979). A review of the hippocampal place cells. Progress in Neurobiology, 13, 419-439.

O'KeEFe, J. (1990). A computational theory of the hippocampal cognitive map. Progress in Brain Research, 83, 301-312.

O'KeEFE, J., * Dostrovsky, J. (1971). The hippocampus as a spatial map: Preliminary evidence from unit activity in the freely moving rat. Brain Research, 34, 171-175.

OKEEFE, J., NADEL, L. (1978). The hippocampus as a cognitive map. New York: Oxford University Press.

Parisi, D., Croconi, F., \& Nolfi, S. (1990). Econets: Neural networks that learn in an environment. Network, 1, 149-168.

RoLLs, E. T. (1989). Functions of neuronal networks in the hippocampus 
and neocortex in memory. In J. H. Byrne \& W. O. Berry (Eds.), Neural models of plasticity (pp. 240-265). San Diego: Academic Press.

Rumelhart, D. E., Zipser, D. (1986). Feature discovery by competitive learning. In D. E. Rumelhart \& J. McClelland (Eds.), Parallel distributed processing: Explorations in the microstructure of cognition (pp. 151-193). Cambridge, MA: MIT Press.

SCHmajuK, N. A. (1990). Role of the hippocampus in temporal and spatial navigation: An adaptive neural network. Behavioral Brain Research, 39, 205-229.

SHARP, P. E. (1991). Computer simulation of hippocampal place cells. Psychobiology, 19, 103-115.

SharP, P. E., Kubie, J. L., * Muller, R. U. (1990). Firing properties of hippocampal neurons in visually symmetrical environments:
Contributions of multiple sensory cues and mnemonic processes. Journal of Neuroscience, 10, 3093-3105.

Tolman, E. C. (1948). Cognitive maps in rats and men. Psychological Review, 55, 189-208.

Treves, A., Rolls, E. T. (in press). Computational constraints suggest the need for two distinct input systems to the hippocampal CA3 network. Hippocampus.

ZIPSER, D. (1985). A computational model of hippocampal place fields. Behavioral Neuroscience, 99, 1006-1018.

(Manuscript received October 18, 1991; revision accepted for publication January 8, 1992.)

\section{Jean S. Morgan 1922-1992}

The Governing Board and the Publications Committee of the Psychonomic Society regret to announce the death of Jean S. Morgan, widow of Clifford T. Morgan, in Austin on February 3, 1992.

Jean Morgan was an integral part of the journal publications operation from its inception in 1964, and was a working member of the Publications Office at the time of her death. Her colleagues at the Publications Office are deeply saddened by this unexpected event, and join the Society's Governing Board and Publications Committee in extending their condolences to her sons, Pete and Michael, and her daughter, Pat, and their families.

For those who may wish to make a contribution in her memory, the Morgan family have suggested the American Red Cross (or Red Cross Disaster Relief Fund (Centex), c/o Hartland Bank, P.O. Box 10994, Austin, TX 78766), the organization in which Jean Morgan was an active volunteer for over 20 years. 